Scientific Visualization, 2020, volume 12, number 1, pages 1 - 9, DOI: 10.26583/sv.12.1.01

\title{
Visualization of EEG signal entropy in schizophrenia
}

\author{
I.E. Kutepov,A, A. V. Krysko,a, V.V. Dobriyan3,A, T.V. Yakovleva4,A, E.Yu. Krylova5,A, \\ V.A. Krysko ${ }^{6, A}$ \\ A Yuri Gagarin State Technical University of Saratov (SSTU) \\ 1 ORCID: 0ooo-0002-1003-4496, iekutepov@gmail.com \\ 2 ORCID: 0000-0002-9389-5602, anton.krysko@gmail.com \\ 3 ORCID: 0000-0003-1136-1867, dobriy88@yandex.ru \\ 4 ORCID: 000o-0003-3238-2317, Yan-tan1987@mail.ru \\ 5 ORCID: 0000-0002-7593-0320, Kat.Krylova@bk.ru \\ 6 ORCID: 0000-0002-4914-764X, tak@san.ru
}

\begin{abstract}
This paper describes the visualization of the study of signal entropy in two groups of subjects. Brain activity signals were obtained using electroencephalogram (EEG). Two groups of adolescents - a schizophrenic group and a control group - were the subjects of the study. For each of the participants in both groups, 16 channels were recorded. Multi-scale entropy, model entropy, and approximated entropy were analyzed for signal complexity. The results of the entropic assessments were compared in the form of topographic images. Topographic images of the head surface were obtained based on a spherical spline. The activity of brain hemispheres for both groups was compared using the mean values of the cross-correlation function.

The study showed that the visualization of EEG signals could be a useful tool for classification of patients with schizophrenia and control groups. The analysis may be considered useful for the psychiatric examination of patients with schizophrenia.

On the other hand, the proposed approach is useful to extend the functionality of the educative robotics. Identification of schizophrenic subjects in the group of students provided by the robotic complex on the fly helps to avoid possible antisocial behavior while applying adequate training methods.
\end{abstract}

Keywords: EEG, schizophrenia, entropy, cross-correlation, data visualization, educational robotics.

\section{Introduction}

Electroencephalogram (EEG) directionally measures the electrical activity of the brain over the surface of the scalp. Modern EEG systems use many electrodes (16-256) to determine the voltage at the corresponding points of the human scalp. Normally, EEG signal sources are not located directly below a particular writing electrode and detectable in a distant region or a deep brain structure at some distance from each of the electrodes. Reverse problem approaches often use numerical models to connect deep, distant sources with the signals recorded on the surface.

This problem can be deemed topographic or the problem of determining the local function of the brain immediately below each writing electrode. The approaches to this problem do not focus on any potential or waveform, but instead, analyze the background EEG activity at each electrode to analyze the frequency spectrum and the characteristics of the oscillation func- 
tion. Instead of simulating a focal source distant from a specific electrode, this approach considers the source ( $\mathrm{s}$ ) below each electrode location [1].

The nature of the voltage measurement requires compliance with the conditions for placing electrodes on the scalp. These conditions include a sufficiently large voltage, sensitivity to brain activity, and the presence of noise [2]. Tentative solutions to this problem include averaging the electrode potential, analyzing bipolar pairs for which the reference effect is obvious, and evaluating the Laplace operator over the surface of the scalp using local or global splines. The averaged potential does not depend on the specific choice of the electrode and at each point approximates the potential relative to infinity. Thus, the approximation of intervening points between the electrodes allows obtaining reliable data on brain activity.

One of the factors affecting the accuracy of topographic analysis results reflecting local brain function is the specific measure of EEG energy selected for mapping. Two most common energy indicators are absolute power or energy intensity at the electrode site in a certain frequency band, measured in $\mathrm{mV}$; and relative power or a fraction of power in the electrode region in a given frequency bandwidth, measured as a percentage of the total absolute power across the spectrum. Previous studies have shown that absolute and relative power are complementary indicators that may transmit significantly different brain function data [3]. The captured EEG data on brain activity allow applying methods of nonlinear dynamics to signals, as well as in other branches of modern science, such as mechanics $[4,5]$, radiophysics [6], history [7], etc.

Time-series entropy analysis is widely used in EEG studies. Multi-Scale Entropy (MSE) analysis was introduced in 2002 to assess the time series complexity by quantifying its entropy at various time scales. The algorithm is successfully used in various fields of research. After its introduction, a number of changes and refinements were proposed, some of which were aimed at improving the accuracy of entropy estimates, while others were aimed at studying alternative methods of analysis. Basic algorithms to determine the entropy are presented in the review [8].

According to literary references, much research is dedicated to the EEG of patients diagnosed with schizophrenia in accordance with various criteria. The most dangerous symptom of schizophrenia is probably a violation of the ambient information perception. Usually, the initial diagnosis of schizophrenia is based on observations of the patient's actions, behavioral changes, familial mental history, a previous level of social functioning, etc. Normally, growth of nonspecific anomalies is reported. The work [9] states that EEG abnormalities and paroxysmal dysrhythmias may have a characteristic effect on the prediction of schizophrenia. Abrams and Taylor [10], using a classification system similar to DSM-IV, showed that patients with schizophrenia had twice as many temporal EEG abnormalities on the left side as patients with affective disorders. Classifying the cases of schizophrenia syndrome based on the analysis of EEG entropy is often a component of machine learning algorithms [11]. In [12], Shannon entropy, spectral entropy, approximated entropy, and the Lempel-Ziv index for the classification of schizophrenia patients were studied. The authors of this work used the visualization of entropy estimates to determine the most representative EEG channels, but they did not consider Multi-Scale entropy. In [13], visualization of spectral entropy was used to show differences in brain activity between schizophrenia patients and the control group.

Interpretation of EEG recordings requires knowledge from four relevant sources of information. Firstly, it takes recognizing and classifying EEG signals with individual identity and temporal patterns that occur when they reappear. Secondly, it is necessary to have a theoretical basis for the signal analysis to facilitate the understanding of both visual and automatic methods. The third requirement relates to the spatial or topographic features of the EEG. The fourth source of information is derived from empirical observations of the relationship between EEG and clinical conditions. Visual recognition of specific EEG samples is much simpler than their oral description because the eyes and brain are particularly good at recognizing images [14]. Mathematical methods of signal analysis allow for quantitative description of EEG recordings, because signal characteristics are measurable. Thus, visualiza- 
tion of EEG signal entropy analysis combines all four sources of information about the subject of the study. The paper introduces an approach to visualize the signal entropy and interrelate the activity of brain zones, i.e. solve the topographic problem and coordinate EEG readings with clinical observations.

In this study, the authors elaborated the examination of the ability of entropy estimates to characterize brain activity zoning in patients with schizophrenia based on topographic images of the head. Three parameters were used to clarify possible differences in the entropy estimation: Multi-Scale Entropy, Sample Entropy, and Approximate Entropy. We analyzed the transmission of information between different regions of the brain cortex both in patients with schizophrenia and in the control group by evaluating the cross-correlation function between the EEG electrodes. This entropy-based visual analysis is considered useful in the psychiatric examination of patients with schizophrenia. In addition, the proposed approach can serve as a tool for the early diagnosis of schizophrenia of students in an educational institution when using robotic systems as a robotic assistant for a teaching professional [24].

\section{Entropy Types}

\subsection{Approximated Entropy (ApEn)}

Pincus recommended an algorithm to determine the approximated entropy [15]. Approximated Entropy (ApEn) is a statistic measure to be used for quantitate measurement of signal complexity or non-regularity [16]. It shows the quantity of fresh information in the signal. A reliable estimate of the approximated entropy can be obtained by analyzing short and noisecontaminated signals. A positive number is assigned to time series with large values, which corresponds to greater complexity or irregularity of the data. Entropy is determined as:

$$
\operatorname{ApEn}(m, r, N)=\varphi^{m}(r)-\varphi^{m+1}(r),
$$

where $m$ is data vector increment, $r$ is phase-space mesh size (error). $\varphi^{m}(r)$ and $\varphi^{m+1}(r)$ components are defined as:

$$
\varphi^{m}(r)=\frac{1}{N-m+1} \sum_{i=1}^{N-m+1} \ln C_{r}^{m}(i),
$$

where $C_{r}^{m}(i)$ is the number of $m$-long interval matches with the error of $r$ across the $N$ data interval.

\subsection{Sample Entropy (SampEn)}

Richman and Moorman developed Sample Entropy in order to eliminate disadvantages of the Approximated Entropy (ApEn) [17]. Approximated Entropy (ApEn) considers signal selfsimilarity. Sample Entropy (SampEn) is the probability that $m$ data sequence coincides with another data sequence for the signal with the error of $r$, that stays the same if the data within the sequence are increased by $m+1$. Sample Entropy (SampEn) is determined from the following formula:

$$
\operatorname{SampEn}(m, r)=\lim _{N \rightarrow \infty}-\ln \frac{A^{m}(r)}{B^{m}(r)},
$$

where $A^{m}(r)$ is the probability of two coinciding datasets for $m+1$ points with error of $r$; $B^{m}(r)$ is the probability of coincidence of two datasets for $m$ points with error of $r$.

Thus, Sample Entropy (SampEn) does not consider self-similarity, avoiding possible $\ln (0)$ problems, with logarithmation at the latest step. SampEn is less dependent on data formatting than ApEn. This makes SampEn algorithm useful for smaller data volume.

\subsection{Multi-Scale Entropy (MSE)}

A method to evaluate Multi-Scale Entropy (MSE) is suggested in the paper [18]. For the given discrete time series $\left\{x_{1}, \ldots, x_{i}, \ldots, x_{N}\right\}$, the sequence is determined from the simplified time series $\left\{y^{(\tau)}\right\}$ regarding the scaling parameter $\tau$. Source time series is divided into $\tau$ long non- 
overlapping windows. The values are averaged for each window afterward. Thus, each element of the simplified time series is determined as:

$$
y_{j}^{(\tau)}=\frac{1}{\tau} \sum_{i=(j-1)_{\tau}+1}^{j r} x_{i}, \quad 1 \leq j \leq N / \tau .
$$

For the first scale, the time series $\left\{y^{(1)}\right\}$ is equivalent to the original time series. The length of each time series corresponds to the length of the original time series divided by the $\tau$ scale parameter.

The $S_{E}$ quantitative measure of entropy for each simplified time series is evaluated as follows:

$$
S_{E}(m, r, N)=\ln \frac{\sum_{i=1}^{N-m} n_{i}^{\prime m}}{\sum_{i=1}^{N-m} n_{i}^{\prime m+1}},
$$

where $m$ is data vector length increment, $r$ is phase-space mesh size (error), $n_{i}^{\prime m}$ is the probability of recurrence of the data sequence having the given length within the input data.

\section{Spherical Spline}

The equations to determine the spherical entropy spline over the skull surface were obtained similarly to the spline of potential presented by Ferry [19].

We assume that the $\bar{r}_{j}$ is the vector to determine the position of the measuring electrode on the spherical scalp surface, at that, $j=1, \ldots, J . V\left(\bar{r}_{j}\right)$ function determines entropy at this point (regarding reference point). Spherical spline to estimate the entropy $V\left(\bar{r}_{j}\right)$ is determined as follows:

$$
V\left(\bar{r}_{j}\right)=c_{0}+\sum_{j=1}^{J} c_{j} g_{m}\left(\rho \cdot \rho_{j}\right),
$$

where $c_{0}$ and $c_{j}$ are data-relevant constants; $\rho \cdot \rho_{j}$ operator is the cosine of the angle between the $\bar{r}$ interpolation point and $\bar{r}_{j}$ electrode position. $g_{m}$ function is determined as follows:

where $P_{n}(x)$ is Legendre's polynomial.

$$
g_{m}(x)=\frac{1}{4 \pi} \sum_{n=1}^{\infty} \frac{2 n+1}{(n(n+1))^{m}} P_{n}(x),
$$

\section{Electrode Layout}

In order to realize the '10-20\%' layout system (Fig. 1) the head size is measured longitudinally from the nasal bridge ('nasion' point) to the inion ('inion' point) and transversely between two auricular tubes. These sizes are taken as 100\% (separately for each direction). Afterward, nominal 'meridians' are drawn from the frontal to the occipital region and transverse 'parallels' through the vertex as a percentage. At the distance of $10 \%$ from the initial points (nasion and inion), the bottom line of electrodes is installed, other electrodes are installed in the points of intersection of 'meridians' and 'parallels' at the distance of $20 \%$ from the full length longitudinally and transversely. Lead points are lettered according to the initial letters of the region names. Left hemisphere points have odd numbers. Midline electrodes are indexed with z (Fz, Cz, Pz) and called sagittal (S - sagittalis). Ear-clip electrodes are lettered with A (A - auriculus; A1, A2). [20] 


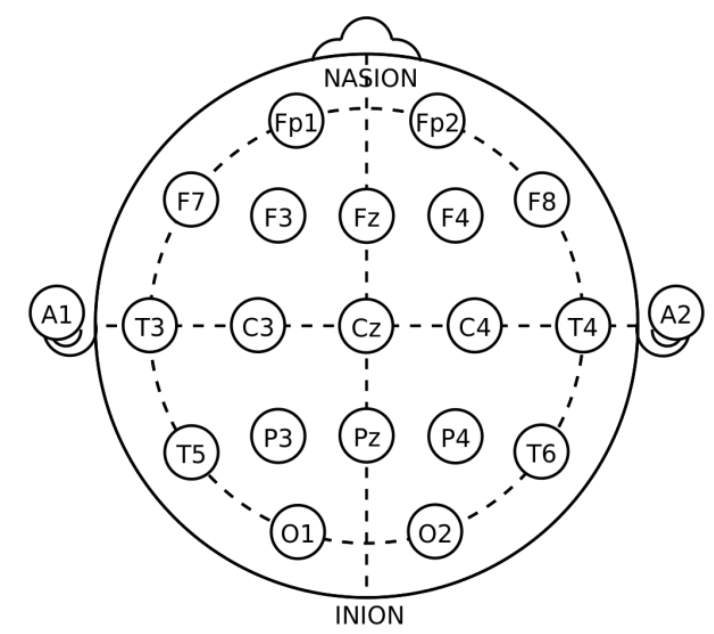

Fig 1. Electrode Installation '10-20\%’ Layout System.

\section{Study Subjects}

The subjects of the study are adolescents at the age of 10 to 14 years. The first group consists of 45 schizophrenic boys at the age of 10 to 14 years, diagnosticated under the criteria listed in [21]. The subjects underwent no medication before the study, so the EEG results may be considered unaffected. The second group included 39 sane boys at the age of 11 to 13 years. EEG of the adolescents is recorded at rest, with eyes closed.

To record EEG, we implemented the '10-20\%' layout system using 16 electrodes: O1, O2, $\mathrm{P}_{3}$, $\mathrm{P}_{4}, \mathrm{Pz}, \mathrm{T} 5, \mathrm{~T} 6, \mathrm{C}_{3}, \mathrm{C} 4, \mathrm{Cz}, \mathrm{T} 3, \mathrm{~T} 4, \mathrm{~F}_{3}, \mathrm{~F} 4, \mathrm{~F} 7, \mathrm{~F} 8$ at the electrode impedance below $10 \mathrm{kOhm}$, sampling rate of $128 \mathrm{~Hz}$, and bandwidth of 0.5 to $45 \mathrm{~Hz}$. Two experts eliminated head and eye motion artifacts manually. The measurement took 60 seconds. EEG source signal database is publicly available online: http://brain.bio.msu.ru/eeg_schizophrenia.htm

\section{Results}

Average entropy in channels for two subject groups is shown in Fig. 2. The following parameter values are used in the calculation: $m=5, r=0.2, \tau=4$. The order of magnitude for the Approximated Entropy (ApEn) is $10^{-3}$, that is characteristic of long signals $N=7680$. The average entropy values for schizophrenic (sch) and control group (norm) subjects are too close to each other, precluding the subject classification. Sample Entropy (SampEn) shows more consistent results as a result of self-similarity consideration. By the way, multiple diagram intersections for schizophrenic (sch) and control group (norm) subjects occur. These intersections disallow using this method for subject classification. Multi-Scale Entropy has shown the best results, having intersections only for O1 channel.
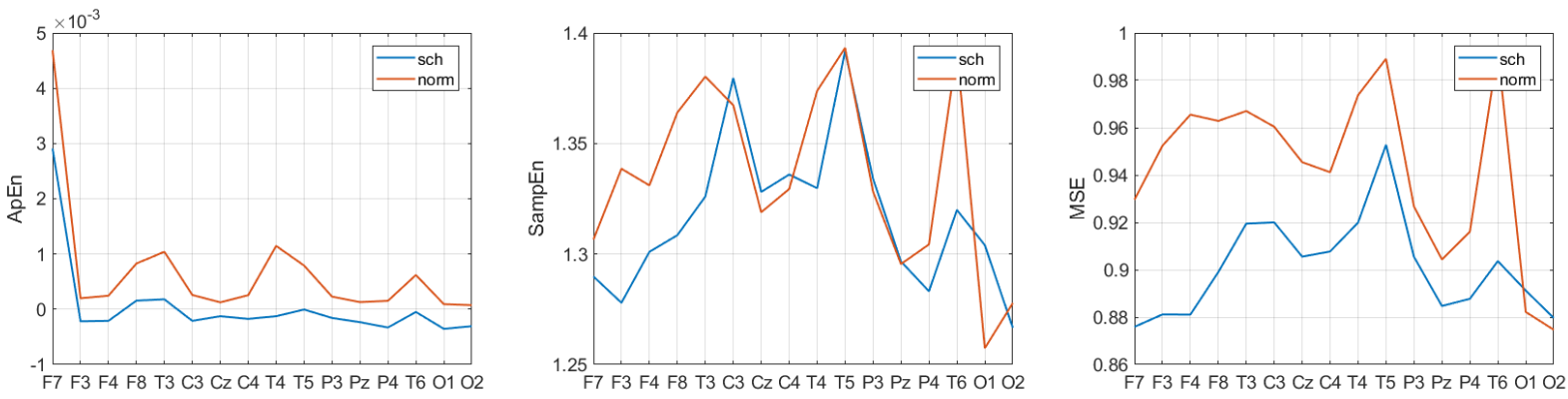

Fig. 2. Channeling of Approximated Entropy (ApEn), Sample Entropy (SampEn) and MultiScale Entropy (MSE).

We used the estimated average entropy values to plot diagrams (Fig. 3) visualizing brain activity determined in the following channels. For the Approximated Entropy (ApEn) (Fig. 2 a) topographic images of schizophrenic subjects and control group are similar and unrepre- 
sentative. For Sample Entropy (SampEn) (Fig. 2 b) and Multi-Scale Entropy (MSE) (Fig. 2 c), topographic images are asymmetric. For schizophrenic subjects, substantial lateralization of brain function is detected in the frontal region of the head. This correlates to other studies [22]. Authors of an MRI-based study also report observable deficiency of brain activity in the left hemisphere of schizophrenic subjects [23]. In our study, we determined hypoactivity in both left and right brain hemispheres, i.e. lowering of the complexity of EEG signals is observed in the entire brain, this representative sign was also mentioned in [12]. Visualization was performed in MatLab software package.

norm

a)

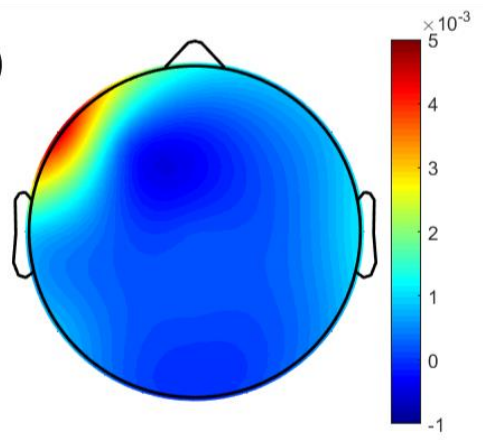

b)

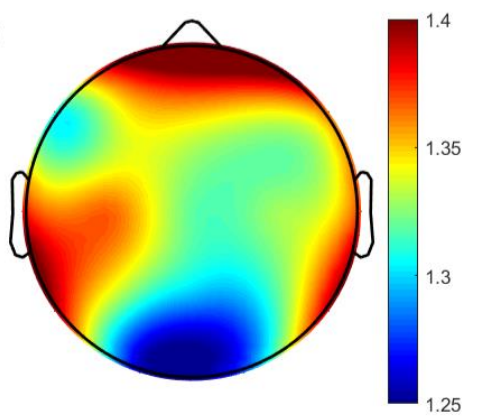

c)

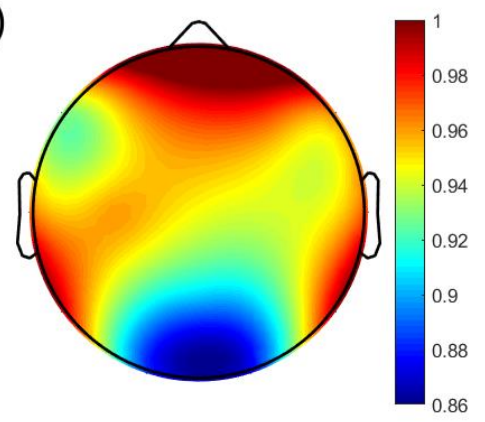

sch
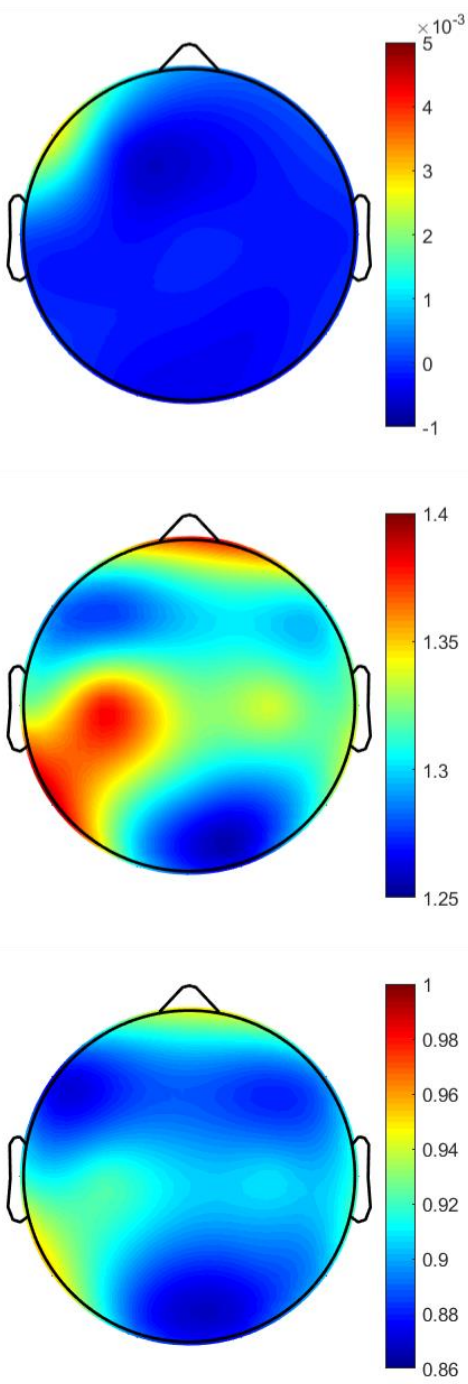

Fig. 3. Topographic Images of the Average Values a) Approximated Entropy (ApEn), b)

Sample Entropy (SampEn) and c) Multi-Scale Entropy (MSE).

Comparison of the images of EEG signal entropy obtained by Multi-Scale Entropy is indicative of the apparent difference between cortical activity in the control group and the schizophrenic subjects. We used the cross-correlation function to estimate this difference and determined the cross-correlation of the subject- and channel-averaged entropy. Fig. 4 shows the visualization of the cross-correlation function for both groups. Numbers (1-16) on the axes correspond to the following channels: $\mathrm{O}_{1}, \mathrm{O}_{2}, \mathrm{P}_{3}, \mathrm{P}_{4}, \mathrm{Pz}_{2}, \mathrm{~T} 5, \mathrm{~T} 6, \mathrm{C}_{3}, \mathrm{C}_{4}, \mathrm{Cz}_{2}, \mathrm{~T}_{3}, \mathrm{~T} 4, \mathrm{~F} 3$, F4, F7, F8. 

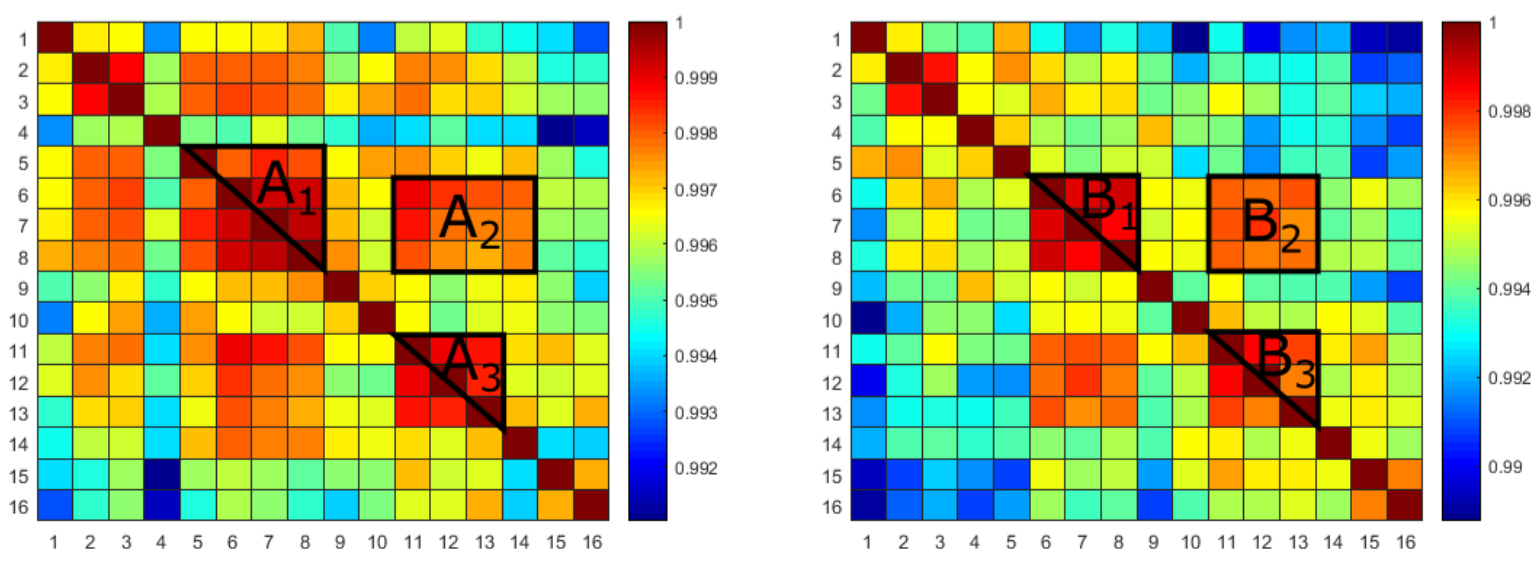

Fig. 4. Cross-Channel Correlation a) In Control Group, b) In Schizophrenic Subjects.

Analysis of the cross-correlation function allowed selection of electrodes with the highest values: 1-4(O1-P4), 4-9(P4-C4), 4-10(P4-Cz), 4-15(P4-F7), 4-16(P4-F8) for control group; 1-7 (O1-T6), 1-12 (O1-T4), 2-10(O2-Cz), 2-12(O2-T4), 2-13(O2-F3), 3-13( $\left.\mathrm{P}_{3}-\mathrm{F}_{3}\right), 3-14\left(\mathrm{P}_{3}-\mathrm{F}_{4}\right), 5^{-}$ 12(Pz-T4), 5-15(Pz-F7), 9-16(C4-F8) for schizophrenic subjects. Thus, lowering of the crosscorrelation entropy in the left hemisphere of the schizophrenic subjects regarding the right and in the left-front brain region regarding right-back one was detected. These results confirm a hypothesis that regional brain activity mismatch may cause schizophrenia. Fig. 4 shows the channel areas with the biggest cross-correlation as $A_{1-3}$ and $B_{1-3}$ for control and schizophrenic groups, respectively. Regions are located similarly, but $\mathrm{A}_{1}$ and $\mathrm{A}_{2}$ dilate regarding $B_{1}$ and $B_{2}$. $A_{1}$ region dilates because of the cross-correlation in the $5-8\left(\mathrm{Pz}-\mathrm{C}_{3}\right), 6-8$ ( $\mathrm{T}_{5}-\mathrm{C}_{3}$ ), and 7-8 (T6- $\left.\mathrm{C}_{3}\right)$ channels, while $\mathrm{A}_{2}$ region dilates because of the cross-correlation in the 6-14 ( $\left.\mathrm{T}_{5}-\mathrm{F}_{4}\right), 7-14\left(\mathrm{~T} 6-\mathrm{F}_{4}\right)$, and 8-14 $\left(\mathrm{C}_{3}-\mathrm{F}_{4}\right)$ channels. Location of the $\mathrm{C}_{3}$ and $\mathrm{F}_{4}$ channels above the left and right brain hemispheres is indicative of the better regional intercommunication in the control group than in the schizophrenic subjects. The results of this study can be used in hardware-software complex of the anthropomorphous robotic assistant for a teaching professional (HSC ARATP) [24] to assess the student's current emotional, psychological and physical condition.

\section{Conclusion}

Authors proposed the visual analysis method combining the advantages of topographical and cross-correlation analysis. This approach allows determining the most representative method (among the studied ones) to evaluate signal complexity and interrelate the activity of the regions and brain regions.

This study has shown that entropy visualization is useful to classify subjects of EEG examination. The most representative results to classify schizophrenic subjects and control group are obtained using Multi-Scale Entropy (MSE) method. Visualization of the average EEG channel entropy gives a metric for lateralization of brain function.

\section{Acknowledgments}

The study is performed with assistance of the RF Ministry of Science and Education (the agreement on providing a subsidy No. 14.577.21.0282 from 1 October 2017, the unique project identifier is RFMEFI57717X0282, the Federal Target Program "Research and Advances on the Priority Directions of Development of a Scientific and Technological Complex of Russia for 2014-2020". 


\section{References}

1. Cook I. A. et al. Assessing the accuracy of topographic EEG mapping for determining local brain function //Electroencephalography and clinical neurophysiology. -1998 . - V. 107. - №. 6. - pp. 408-414.

2. Nunez P. L. et al. Electric fields of the brain: the neurophysics of EEG. - Oxford University Press, USA, 2006.

3. Leuchter A. F. et al. Regional differences in brain electrical activity in dementia: use of spectral power and spectral ratio measures //Electroencephalography and Clinical Neurophysiology. - 1993. - V. 87. - №. 6. - pp. 385-393.

4. J. Awrejcewicz, V. A. Krysko, I. V. Papkova, A. V. Krysko Deterministic Chaos in OneDimensional Continuous Systems. World Scientific, Singapore 2016 monograph, 562 pages, ISBN978-981-4719-69-8

5. Krysko A.V., Awrejcewicz J., Zakharova A.A., Papkova I.V., Krysko V.A., 'Chaotic vibrations of flexible shallow axially symmetric shells', Nonlinear Dynamics, 91(4), 2018, 2271-2291

6. Stankevich N.V., Dvorak A.A., Astakhov V.V., Jaros P., Perlikowski P., Kapitaniak T., Kapitaniak M. Chaos and hyperchaos in coupled antiphase driven Toda oscillators. Regular and Chaotic Dynamics. 2018. V. 23. № 1. pp. 120-126.

7. Yaroshenko, T.Y., D.V. Krysko, V. Dobriyana, M.V. Zhigalova, H. Vos, P. Vandenabeele, and V.A. Krysko. 2015. Wavelet Modeling and Prediction of the Stability of States: The Roman Empire and the European Union. Communications in Nonlinear Science and Numerical Simulation 26 (1-3): 265-275.

8. Humeau-Heurtier A. The multiscale entropy algorithm and its variants: A review //Entropy. - 2015. - V. 17. - №. 5. - pp. 3110-3123.

9. Henry J. C. Electroencephalography: basic principles, clinical applications, and related fields //Neurology. - 2006. - V. 67. - №. 11. - pp. 2092-2092-a.

10. Abrams R., Taylor M. A. Differential EEG patterns in affective disorder and schizophrenia //Archives of General Psychiatry. - 1979. - T. 36. - №. 12. - pp. 13551358.

11. Piryatinska A., Darkhovsky B., Kaplan A. Binary classification of multichannel-EEG records based on the $\epsilon$-complexity of continuous vector functions //Computer methods and programs in biomedicine. - 2017. - V. 152. - pp. 131-139.

12. Sabeti M., Katebi S., Boostani R. Entropy and complexity measures for EEG signal classification of schizophrenic and control participants //Artificial intelligence in medicine. - 2009. - V. 47. - №. 3. - pp. 263-274.

13. Bachiller A. et al. Decreased spectral entropy modulation in patients with schizophrenia during a P300 task //European archives of psychiatry and clinical neuroscience. - 2014. - V. 264. - №. 6. - pp. 533-543.

14. Cooper R., Osselton J. W., Shaw J. C. EEG technology. - Butterworth-Heinemann, 2014.

15. Pincus S. M. Approximate entropy as a measure of system complexity //Proceedings of the National Academy of Sciences. - 1991. - V. 88. - №. 6. - pp. 2297-2301.

16. Bruhn J., Röpcke H., Hoeft A. Approximate entropy as an electroencephalographic measure of anesthetic drug effect during desflurane anesthesia //Anesthesiology: The Journal of the American Society of Anesthesiologists. - 2000. - V. 92. - №. 3. - pp. 715-726.

17. Richman J. S., Moorman J. R. Physiological time-series analysis using approximate entropy and sample entropy //American Journal of Physiology-Heart and Circulatory Physiology. - 2000. - V. 278. - №. 6. - pp. H2039-H2049.

18. Costa M., Goldberger A. L., Peng C. K. Multiscale entropy analysis of biological signals //Physical review E. - 2005. - V. 71. - №. 2. - pp. 021906. 
19. Ferree T. C. Spherical splines and average referencing in scalp electroencephalography //Brain topography. - 2006. - V. 19. - №. 1-2. - pp. 43-52.

20. Klem G. H. et al. The ten-twenty electrode system of the International Federation //Electroencephalogr Clin Neurophysiol. - 1999. - V. 52. - №. 3. - pp. 3-6.

21. Md W. M. Diagnostic and statistical manual of mental disorders. - 1994.

22. Na S. H. et al. EEG in schizophrenic patients: mutual information analysis //Clinical Neurophysiology. - 2002. - V. 113. - №. 12. - pp. 1954-1960.

23. Hugdahl K. et al. Brain activation measured with fMRI during a mental arithmetic task in schizophrenia and major depression //American Journal of Psychiatry. 2004. - V. 161. - №. 2. - pp. 286-293.

24. Stepanov M.F. et al. Architectural features of a cyber-physical control system of hardware-software complex of anthropomorphous robot assistant for a teaching professional // Mathematical Methods in Technics and Technologies (MMTT - 32). Proceedings of 32th International Conference. - SPb.: Peter the Great St. Petersburg Polytechnic University, 2019. - pp. 26 - 36. 